\title{
ENERGY-DISPERSIVE X-RAY REFLECTOMETRY AND X-RAY GRAZING INCIDENCE DIFFRACTION FROM ORGANIC MULTILAYERS
}

\author{
F. Neißendorfer, A. Bolm and U. Pietsch \\ Institute of Solid State Physics, University of Potsdam, 14469 Potsdam, Germany
}

\begin{abstract}
The installation of the wavelength shifter at the BESSY I storage ring in Berlin makes it possible to apply the synchrotron radiation for white beam investigations of organic multilayers. Considering the energy characteristic of the synchroton radiation source and the absorbance of the beryllium window the synchrotron radiation can be used outside the UHV system for X-ray reflectometry and X-ray diffuse scattering between about $3 \mathrm{keV}$ and $25 \mathrm{keV}$. Between 3 and $10 \mathrm{keV}$ the synchrotron radiation intensity is high enough to realize the grazing incidence diffraction mode in order to get in-plane information. The capability of the methods is demonstrated at the example of a $\mathrm{Pb}$-stearate multilayer covered by a thin polyelectrolytic polymer layer.

PACS numbers: $61.10 . \mathrm{Kw}, 68.55 . \mathrm{Jk}, 68.18 .+\mathrm{p}, 07.85 . \mathrm{Qe}$
\end{abstract}

\section{Introduction}

X-ray reflectometry (XSR) is widely accepted for nondestructive investigation of lamellarly structurized organic multilayers. Using an energy-dispersive setup (EDS) the whole reflectivity pattern can be recorded simultaneously. In this case a well-collimated white beam strikes the sample surface under a fixed angle of incidence $\alpha_{\mathrm{i}}$ and the reflected beam is recorded by an energy dispersive detection system at $2 \alpha_{\mathrm{i}}$. The higher signal to noise ratio and the constant sample area which is under investigation are additional advantages of this technique [1]. It has been recently shown that organic multilayers built by fatty acid salts molecules are not uniform [2]. The films consist of domains with an extension of several $100 \mathrm{~nm}$ separated by deep holes and scratches. Information about the lateral domain size is a vailable via X-ray diffuse scattering (XDS) [3]. The crystalline order of the molecular arrangement within the domains can be verified using $\mathrm{X}$-ray grazing incidence diffraction (GID) $[4,5]$. Due to the powder-like distribution of crystalline domains the diffracted intensity becomes available rotating the detector around the surface normal. Because of the very low intensity of these in-plane Bragg reflections $\left(R \approx 10^{-8}\right)$ the GID mode could only be realized at high power synchrotron radiation (SR) sources using angular dispersive equipments. GID measurements by EDS are still missing. Its first realization is demonstrated in this note. 


\section{Sample preparation}

The sample was prepared by combining the Langmuir-Blodgett and the dipping technique. The details of this procedure are extensively described elsewhere [6]. Eleven monolayers of $\mathrm{Pb}$-stearate were transferred on a silicon support. After the complete exchange of the subphase one bilayer of DODAB (dimethyldioctadecylamonium bromide) and PSS (polystyrenesulfonate) was deposited on top of the multilayer. Then the wafer was dipped alternatively into a solution of PAH (polyallylamine hydrochloride) and PSS. In all two double layers of PAH/PSS are grown on top of the $\mathrm{Pb}$-stearate multilayer which corresponds to a top layer thickness of about $20 \mathrm{~nm}$.

\section{The energy-dispersive reflectometry at BESSY I}

A self-equipped reflectometer was installed at the WLS beam line 21.30 at BESSY I last year [7]. The white SR beam leaves the UHV system of BESSY across a $25 \mu \mathrm{m}$ beryllium window protected in a He-stream. Thus the experiment can be performed under air condition using a Huber goniometer equipped on a $z$-stage. The reflected intensity is recorded by a $\mathrm{Si}(\mathrm{Li})$ detector having an energy resolution of about $160 \mathrm{eV}$ at $5 \mathrm{keV}$. The high power SR is controlled via a step motor driven incident slit and using thin aluminium absorber foils in front of the detector window. The whole equipment is inhoused by a commercial radiation shield.

White synchrotron radiation (SR) can be used within an energy window between 2 and $35 \mathrm{keV}$. Due to the exponential decay of SR intensity for increased e.ergies and the absorbance of the beryllium and aluminium foils maximum intensity is found close to $5 \mathrm{keV}$. Here, the detection efficiency is rather limited by the saturation of the detector system than by the reflectivity of the sample used. Thus the available reflectivity spectrum has to becorded in two steps: first, at low energies with very narrow incident slits and using a very thin aluminium foil and

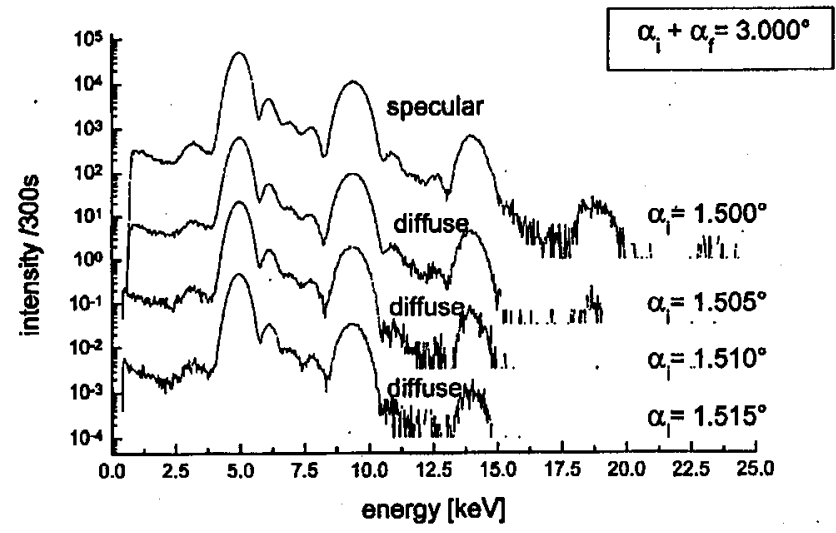

Fig. 1. Energy dispersive specular reflectivity and X-ray diffuse scattering for $\alpha_{\mathrm{i}}+\alpha_{\mathrm{f}}=3^{\circ}$. 
second, for larger energies using higher incident intensity but a $1 \mathrm{~mm}$ aluminium plate to protect the detector and to cut the most intensive SR at lower energies. Figure 1 shows the XSR spectrum recorded from sample 1 at $\alpha_{i}=3.0^{\circ}$. Five Bragg maxima appear at

$$
E_{n}=12.4 /\left[d\left(\alpha_{\mathrm{i}}+\alpha_{\mathrm{f}}\right)\right] .
$$

The energy separation $E_{n+1}-E_{n}$ measures the vertical spacing of the multilayer of $d \approx 5 \mathrm{~nm}$ which is in agreement with the literature [5]. Note that the edge of total external reflection is invisible $\left(E_{\mathrm{c}} \leq 2 \mathrm{keV}\right)$. Figure 1 shows several XDS spectra in addition. They were accumulated at fixed $\alpha_{\mathrm{i}}+\alpha_{\mathrm{f}}\left(\alpha_{\mathrm{f}}-\right.$ exit angle) but changing $\alpha_{i}$ and contain similar information as "longitudinal-diffuse" scans recorded by an angular dispersive scan. For definite $E_{n}$ but varying $\alpha_{i}$ the change of intensity corresponds to a "transversal-diffuse" scan in the angular dispersive mode. As demonstrated by Mahler et al. [8] the diffuse intensity increases if the lateral domain size is decreased and vice versa. Thus energy-dispersive XDS can be used to probe the kinetics of the domain melting process under thermal annealing.

\section{The energy-dispersive grazing-incidence diffraction}

The 2D-crystalline order of fatty acid salt molecules can be evaluated using GID. Taking into account the energy characteristic of the SR source the detector has to be displaced by about $2 \Theta_{\text {in }} \approx 30^{\circ}$ away from the incidence plane in order to fix the most intensive Bragg peaks between 3 and $10 \mathrm{keV}$. Beside the incidence intensity $I_{0}(E)$ the expected Bragg intensity is proportional to

$$
I(E) \approx I_{0}(E)\left|T\left(\alpha_{\mathrm{i}}\right) T\left(\alpha_{\mathrm{f}}\right) F(h k)\right|^{2} .
$$

The amount of the Fresnel transmission functions $T\left(\alpha_{i, f}\right)$ depends on the energy again

$$
T\left(\alpha_{\mathrm{i}, \mathrm{f}}\right)=\frac{2 \alpha_{\mathrm{i}, \mathrm{f}}}{\alpha_{\mathrm{i}, \mathrm{f}}+\left[\left(\alpha_{\mathrm{i}, \mathrm{f}}^{2}-\alpha_{\mathrm{c}}^{2}(E)\right]^{1 / 2}\right.} .
$$

$F(h k)$ is the 2D structure factor. Figure 2 (top curve) displays a diffraction spectrum recorded at $2 \Theta_{\text {in }} \approx 33^{\circ}$ and $\alpha_{\mathrm{i}}=0.32^{\circ}$. It shows several intensive peaks on the background of the incident SR spectrum. Those peaks which belong to the in-plane structure of our sample can be identified recording an air-scattering spectrum without the sample (see Fig. 2 below). The peaks at $7.5 \mathrm{keV}$ and about $8 \mathrm{keV}$ are Ni-fluorescence lines, as example. All the spectra were recorded during $300 \mathrm{~s}$ using slit widths of about $0.1 \times 5 \mathrm{~mm}^{2}$ (incident side) and $0.2 \times 9.5 \mathrm{~mm}^{2}$ (detector side) under single bunch conditions. The sample information can be separated from the incident spectrum dividing the sample spectrum by the spectrum of air scattering. Figure 3 shows several spectra recorded at different $\alpha_{i}$ and normalized by the named procedure. Up to 5 Bragg peaks appear. Their energies found at $E \approx 5.3,6.0,8.8,10.0$ and $10.7 \mathrm{keV}$ correspond to lattice parameters of 4.10 , $3.65,2.48,2.18$ and $2.07 \AA$. These values are in excellent agreement with those of angular dispersive measurements. They describe the (11), (02), (20), (13) and the (22) reflections of a distorted orthorhombic 2D lattice [4]. This means that the in-plane structure of our $\mathrm{Pb}$-stearate multilayer is similar to a respective film without covering by a polyelectrolyte top layer. 


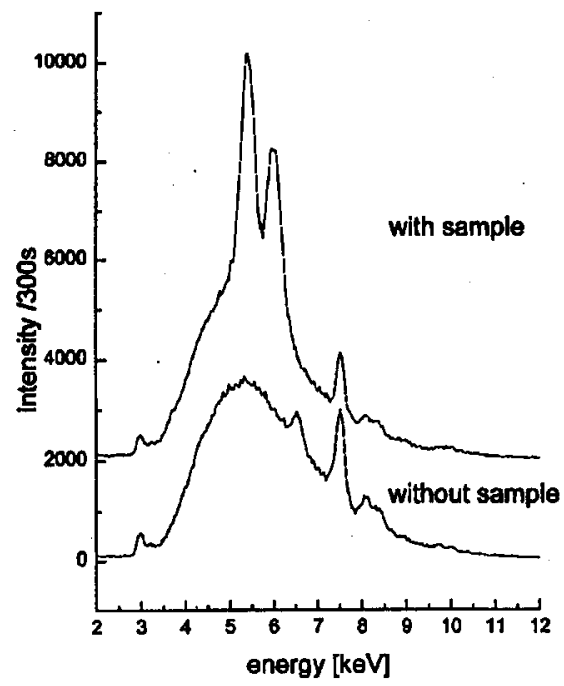

Fig. 2. In-plane diffraction spectrum recorded at $2 \Theta=33^{\circ}$ with and without the sample.

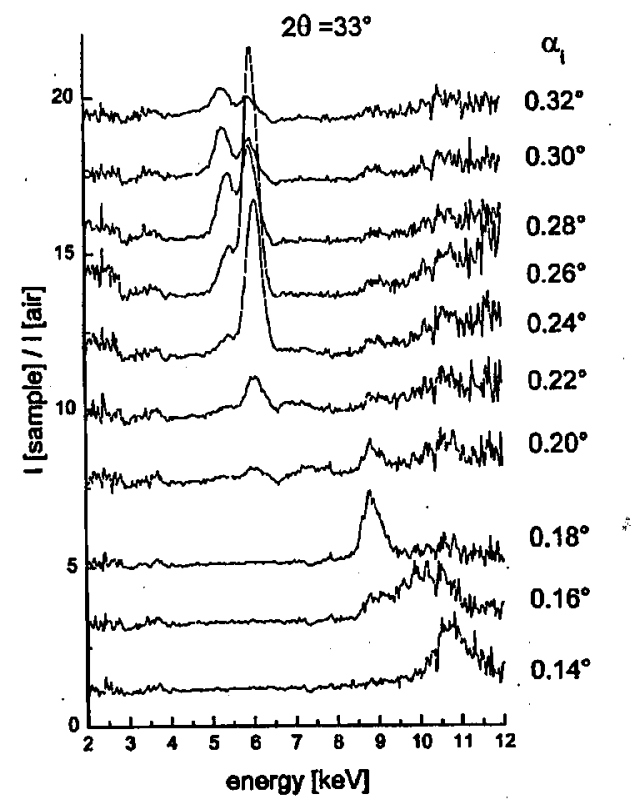

Fig. 3. Normalized in-plane diffraction spectra recorded at different $\alpha_{i}$. For more clarity the spectra are spaced vertically.

As shown in Fig. 3 in addition, the Bragg peaks become maximum at different $\alpha_{i}$. This is due to the energy dependence of $\alpha_{c}$ which varies $T\left(\alpha_{i, f}\right)$ given in Eq. (3). 
For a separate measurement of a single Bragg peak we have to optimize both $\alpha_{i}$ and $\alpha_{\mathrm{f}}$.

In summary, the EDS was successfully applied to record reflectometry and GID spectra from an organic multilayer. Because the whole scattering curve appears simultaneously and due to the high intensity of SR the EDS is suited for time resolved measurements. Under the concrete conditions of BESSY I the minimum time to record one spectrum can be reduced down to 15 seconds [7].

\section{Acknowledgments}

The authors thank Mr. Frank Essler (University of Mainz) for his help during the sample preparation and H. Möhwald (MPI-KGF) and W. Gudat (BESSY Berlin) for the continuous interest in this experiment.

\section{References}

[1] T.H. Metzger, C. Luidl, U. Pietsch, U. Vierl, Nucl. Instrum. Methods Phys. Res. A 350, 398 (1994).

[2] U. Pietsch, T.A. Barberka, U. Englisch, R. Stömmer, Thin Solid Films 284-285, 387 (1996).

[3] R. Stömmer, U. Englisch, U. Pietsch, V. Holy, Physica B 221, 284 (1996).

[4] T.A. Barberka, private communication.

[5] T.A. Barberka, Ph.D. Thesis, Potsdam 1996.

[6] F. Essler, Diploma work, Universität Mainz, 1995.

[7] F. Neißendorfer, U. Pietsch, H. Möhwald, BESSY Annual Report 1995, p. 515.

[8] W. Mahler, T.A. Barberka, U. Pietsch, U. Höhne, H.J. Merle, Thin Solid Films 256, 198 (1995). 